\title{
Endocarditis associated with Chlamydia trachomatis infection
}

\author{
B F BREARLEY, D N HUTCHINSON
}

From the Royal Infirmary, Preston, Lancs

SUMMARY A 43-year-old man with a ventricular septal defect presented with pyrexia of unknown origin. Routine blood cultures were negative but serological evidence of infection with Chlamydia trachomatis was obtained.

It is recognised that Chlamydia psittaci infection may involve the heart, causing myocarditis, pericarditis, ${ }^{1}$ and possibly valvular disease. ${ }^{2}$ Its role as the causative agent in endocarditis ${ }^{34}$ has been less commonly reported. More recently, endocarditis caused by $C$. trachomatis infection has been described. ${ }^{5}$ Most of the infections have involved one or more heart valves in patients with or without a history of rheumatic fever. We describe the development of endocarditis in association with C. trachomatis in a patient with a congenital ventricular septal defect and no evidence of valvular disease.

\section{Case report}

A 43-year-old schoolteacher was admitted to hospital with a one month history of a remitting influenza-like illness, with night sweats and a flitting polyarthropathy involving shoulders, knees, hips, and elbows. His appetite had been poor and he had lost $3 \mathrm{~kg}$ in weight. Twenty-four hours before admission he had developed a swollen and painful left ankle.

The patient was found to be pyrexial $\left(39.8^{\circ} \mathrm{C}\right)$ and had a mild conjunctivitis. A pansystolic murmur was audible all over the chest, being maximal at the left sternal edge. The left ankle was swollen and felt hot, with the skin over the joint being erythematous; there was almost total restriction of movement of the joint. Apart from a minor degree of limitation of hip movement-which had been present for some years previously-other joints were not affected. A provisional diagnosis of rheumatic fever was made.

The haemoglobin level was $12.2 \mathrm{~g} / \mathrm{dl}$ and the white blood cell count $7 \cdot 7 \times 10^{9} / 1$. The erythrocyte sedimentation rate was raised (41 $\mathrm{mm}$ in first hour). Microscopical and cultural examination of urine was negative. Urea, electrolytes, protein, and liver function tests were normal. Antistreptolysin-0 titre, brucella agglutination test, rheumatoid factor, and autoantibody screens were negative. A throat swab grew normal upper respiratory flora. Two blood cultures taken on and shortly after admission remained sterile. The tuberculin test was weakly positive while radiographic examination of the chest did not disclose any abnormality. The electrocardiogram was normal. M-mode and cross-sectional (2D) echocardiograms indicated normal aortic, mitral, pulmonary, and tricuspid valves, and no chamber enlargement. The features were compatible with a small ventricular septal defect ( $\mathrm{Dr}$ A K Brown, Lancaster). Blood samples collected approximately 30,43 , and 63 days after the onset of the illness gave titres of 10,20 , and 320 , respectively, to the chlamydial group complement fixing antigen.

With the diagnosis of rheumatic fever in mind, the patient was treated with aspirin and started on phenoxymethyl penicillin, after which his temperature settled within 24 hours. On further examination, however, splinter haemorrhages were seen and the antibiotic was discontinued after five doses had been given. His pyrexia returned after four days without treatment and six further blood cultures over the next 12 days remained sterile. In view of his persisting fever, however, it was decided to restart antibiotic treatment, and the patient was given $3 \mathrm{~g}$ benzyl penicillin every six hours by intravenous bolus injection. His temperature settled within 48 hours and remained normal. The chlamydial infection was reported within a week of the patient starting intravenous treatment, and as he was responding satisfactorily it was decided to continue with the pencillin. Unfortunately, after 17 days of treatment he again became pyrexial and developed a maculopapular eruption on his chest and abdomen. 
Antibiotic treatment was stopped for 36 hours and the rash and pyrexia settled. Erythromycin $600 \mathrm{mg}$ intravenously thrice daily was started and continued for 14 days, thereafter being given orally $(500 \mathrm{mg}$ q.d.s.) for a further three weeks.

Detailed questioning of the patient failed to disclose even distant contact with birds or animals. The sera were therefore sent to Dr P J L Sequeira (Central Serology Laboratory, Withington Hospital, Manchester) who examined them by the microimmunofluoresence technique for antibody to C. trachomatis. The results (Table) were reported

Table Chlamydia microimmunofluorescence tests (titres)

\begin{tabular}{llll}
\hline Days after onset & 39 & 43 & 63 \\
\hline Hyperendemic pool* & $\mathrm{Neg}$ & $\mathrm{Neg}$ & $\mathrm{Neg}$ \\
Genital pool* $_{\text {LGV pool* }}^{*}$ & 32 & 32 & 512 \\
Human ocular & 32 & 32 & 128 \\
\hline
\end{tabular}

*Tests for specific IgM to the pooled antigen were included but were negative in each group.

as being consistent with an infection with an oculogenital strain of $C$. trachomatis. The gonococcal complement fixation test was negative.

\section{Comment}

This patient illustrates the problem of management of a subject with signs and symptoms of bacterial endocarditis but negative blood cultures. In this instance giving an antibiotic to the patient after collection of only two blood cultures increased the difficulties. Blood cultures, collected up to 16 days after the last of five doses of antibiotic, remained sterile; this suggested that an uncommon agent was present, though there is a report of blood cultures remaining negative for 17 days after a single dose of penicillin. ${ }^{6}$ A recent leading article ${ }^{7}$ discusses the possible aetiological agents and recommends a combination of penicillin and gentamicin treatment for patients with infective endocarditis who present with repeatedly negative blood cultures. In this instance, though penicillin alone was used, the initial response was excellent. If the switch to erythromycin had not been rendered necessary because of allergy, a course of tetracycline or erythromycin after the penicillin would have been advisable, as in vitro studies using $C$. psittaci chronically infected tissue cultures have shown that penicillin suppresses but does not eradicate infection. ${ }^{8}$

Until recently, in chlamydial endocarditis conclusive evidence of the species of the infecting organism has only been achieved by demonstration of the agent in damaged valves removed at operation or at necropsy. The development of the microimmunofluorescence test ${ }^{\circ}$ for the detection of antibody to $C$. trachomatis has made it more possible to differentiate infection with $C$. psittaci and $C$. trachomatis by serological methods. The failure in this patient to show the presence of specific IgM does not exclude the diagnosis of infection with C. trachomatis. The detection of a low unchanging antibody level in the first two sera suggests residual antibody from a previous infection with $C$. trachomatis, and therefore a specific IgM response would not be expected. Moreover, the possible presence of residual chlamydial antibody makes it imperative that a fourfold rise in titre is obtained before serological tests alone can be considered significant.

It is our opinion that $C$. trachomatis should be considered as a cause of bacterial endocarditis in patients with negative blood cultures and that serological studies in those subjects showing a rising chlamydial complement fixation titre should be extended to include chlamydial microimmunofluorescence tests.

\section{References}

1 Sutton GC, Morrissey RA, Tobin JR Jr, Anderson TO. Pericardial and myocardial disease associated with serological evidence of infection by agents of the Psittacosis-Lymphogranuloma Venereum group (Chlamydiaceae). Circulation 1967; 36: 830-8.

2 Ward C, Ward AM. Acquired valvular heart-disease in patients who keep pet birds. Lancet 1974 ; ii: 734-6.

3 Levison DA, Guthrie W, Ward C, Green DM, Robertson PGC. Infective endocarditis as part of Psittacosis. Lancet 1971; ii: 844-7.

4 Birkhead JS, Apostolov K. Endocarditis caused by a psittacosis agent. Br Heart $\mathcal{f}$ 1974; 36: 728-31.

5 van der Bel-Kahn JM, Watanakunakorn C, Menefee MG, Long HD, Dicter R. Chlamydia trachomatis endocarditis. Am Heart $\mathcal{F}$ 1978; 95: 627-36.

6 Balme HW, Dormer AE. Bacteriostatic agents in the treatment of subacute bacterial endocarditis. $\mathrm{Br} \mathrm{Med}$ f 1954; 1: 500-1.

7 Anonymous. Infective endocarditis with negative blood cultures (leading article). $\mathrm{Br} \operatorname{Med} \mathcal{F} 1979$; ii: 4.

8 Galasso GJ, Manire GP. Effect of antiserum and antibiotics on persistent infection of HeLa cells with meningopneumonitis virus. $\mathcal{f}$ Immunol 1961; 86: 382-5.

9 Wang S, Grayston JT. Human serology in Chlamydia trachomatis infection with microimmunofluorescence. f Infect Dis 1974; 130: 388-97.

Requests for reprints to Dr D N Hutchinson, The District Laboratory, Royal Infirmary, Preston PR1 6PS, Lancashire. 